\title{
Suction/Inspiration against resistance or standardized Mueller maneuver : a new breathing technique to improve contrast density within the pulmonary artery: a pilot $\mathrm{CT}$ study
}

\author{
Andreas Gutzeit ${ }^{1,2,3}$ • Johannes M. Froehlich ${ }^{2} \cdot$ Stephan Wälti $^{4}$ - Justus E. Roos ${ }^{5}$. \\ Matthias Meissnitzer $^{1} \cdot$ Klaus Hergan $^{1} \cdot$ Constantin von Weymarn $^{2} \cdot$ David Czell $^{6}$. \\ Matthias Goyen $^{2} \cdot$ Carolin Reischauer ${ }^{1,2}$
}

Received: 3 December 2014 /Revised: 18 February 2015 / Accepted: 23 March 2015 / Published online: 2 June 2015

(C) European Society of Radiology 2015

\begin{abstract}
Objective Our aim was to prospectively investigate whether the recently introduced suction/inspiration against resistance breathing method leads to higher computed tomography (CT) contrast density in the pulmonary artery compared to standard breathing.

Material and methods The present study was approved by the Medical Ethics committee and all subjects gave written informed consent. Fifteen patients, each without suspicious lung emboli, were randomly assigned to four different groups with different breathing maneuvers (suction against resistance, Valsalva, inspiration, expiration) during routine CT. Contrast enhancement in the central and peripheral sections of the pulmonary artery were measured and compared with one another.
\end{abstract}

Electronic supplementary material The online version of this article (doi:10.1007/s00330-015-3735-y) contains supplementary material, which is available to authorized users.

Andreas Gutzeit

agutzeit2000@gmail.com

1 Department of Radiology, Paracelsus Medical University Salzburg, Salzburg, Austria

2 Institute of Radiology and Nuclear Medicine, Clinical Research Unit, Hirslanden Hospital St. Anna, St. Anna Strasse 12,

6006 Lucerne, Switzerland

3 Department of Radiology, Kantonsspital Winterthur, Brauerstrasse 15, 8401 Winterthur, Switzerland

4 Department of Radiology and Nuclear Medicine, Cantonal Hospital St. Gallen, 9007 St. Gallen, Switzerland

5 Department of Radiology, Duke University Medical Center, Durham, NC, USA

6 Department of Neurology, Cantonal Hospital Winterthur, Brauerstrasse 15, 8401 Winterthur, Switzerland
Results Peripheral enhancement during suction yielded increased mean densities of 138.14 Hounsfield units (HU) $(p=$ 0.001), compared to Valsalva and a mean density of $67.97 \mathrm{HU}$ superior to inspiration $(p=0.075)$. Finally, suction in comparison to expiration resulted in a mean increase of $30.51 \mathrm{HU}(p=$ 0.42). Central parts of pulmonary arteries presented significantly increased enhancement values $(95.74 \mathrm{HU})$ for suction versus the Valsalva technique $(p=0.020)$, while all other mean densities were in favour of suction (versus inspiration: $p=$ 0.201 ; versus expiration: $p=0.790$ ) without reaching significance.

Conclusion Suction/Inspiration against resistance is a promising technique to improve contrast density within pulmonary vessels, especially in the peripheral parts, in comparison to other breathing maneuvers.

Key Points

- Suction/Inspiration against resistance is promising to improve contrast density within the pulmonary artery.

- Patients potentially suffering pulmonary embolism are able to follow suction/inspiration against resistance.

- Contrast density after suction is superior in comparison to other breathing maneuvers.

Keywords Breathing · Pulmonary embolism · Computed tomography angiography $\cdot$ Pulmonary vessel $\cdot$ Contrast density

\section{Introduction}

Diagnosing pulmonary embolism (PE) is a common medical problem in daily radiological practice. The incidence of $\mathrm{PE}$ is approximately 60 to 70 per 100,000 in the general western population. In the case of known venous thrombosis, 
approximately 124 per 100,000 of the general population develop PE [1]. The real number of PE is probably underestimated and there seems to be a high number of unexpected cases of PE. Approximately 39.5 to $49.5 \%$ of patients with deep vein thrombosis (DVT) without any symptoms develop PE [2]. In postmortem autopsy investigations, it could be shown that PE had not been diagnosed before death in over approximately $45 \%$ of these retrospectively analyzed patients [3].

Contrast-enhanced computed tomography (CT) angiography is the internationally accepted gold standard for the examination of patients with suspected pulmonary embolism [4].

Summarizing international studies, the sensitivity of CT angiography for correctly diagnosing PE is reported as lying between $53 \%$ and $100 \%$, with specificities between $83 \%$ and $100 \%[5,6]$. It is a generally well-known radiological challenge and a real problem in daily routine that contrast enhanced PE CTs suffer from weak or insufficient contrast within the pulmonary artery (PA). The problem of insufficient contrast enhancement in the PA might be that examinations can mimic a false-positive embolus or that relevant emboli are overlooked [7].

Various techniques, modern contrast media and varying iodine concentrations, as well as optimization of contrast administration schemes, are all being discussed with the final goal of overcoming the above-mentioned diagnostic constraints.

A rarely addressed issue concerns the influence of various breathing techniques during the injection of the contrast agent on image enhancement properties and on diagnostic accuracy. There is an ongoing debate regarding what might be the best patient breathing method to improve the image quality and enhancement profile of pulmonary CT angiography [8-10].

In order to obtain optimal contrast, as much contrastenhanced blood as possible should arrive from the superior vena cava while injecting the contrast agent via the upper extremity (regular intravenous access via dorsal hand or cubital vein). Moreover, a rather small amount of noncontrasted blood should run from the inferior vena cava (IVC) into the right atrium. It is well known that noncontrasted blood originating from the abdominal organs and lower extremities via the IVC influences the dilution of contrast medium in the heart and finally in the PA. This phenomenon is called "transient interruption" of contrast bolus, and might be the reason for weak contrast in a multitude of routine situations [10].

In the last few years, some research groups have postulated that "expiration" followed by apnea during injection of the contrast agent might improve the enhancement profile within the PA CT [8-13].

A recently published magnetic resonance (MR) study with volunteers demonstrated for the first time that a Mueller-like maneuver (suction or inspiration against resistance) could significantly elevate blood flow through the SVC while reducing blood flow through the IVC [14]. Nevertheless, it is not known if this observation has any relevant clinical influence or if truly guarantees better contrast within the PA of patients during contrast-enhanced CT.

Thus, the aim of the present study was to clarify whether the new standardized breathing method of "suction or inspiration against resistance or standardized Mueller maneuver" is able to improve the contrast density within the PA during a standardized PA CT in comparison to other breathing methods.

\section{Material and methods}

A cohort of 148 patients scheduled for a thorax CT examination without clinical suspicion of PE was prospectively enrolled between February 2013 and December 2013. All patients were asked for inclusion accepting a standardized lung-emboli CT before routine venous CT. Exclusion criteria were reduced renal function (11 patients), known chronic obstructive pulmonary disease (COPD) (18 patients), mental or psychological retardation (five patients), problems in understanding the local language (19 patients) and patients with lung cancer or lung metastases (35 patients). This study was approved by the institutional review board and all patients gave written informed consent.

The remaining 60 patients were randomly divided into four groups of 15 patients, each undergoing different breathing maneuvers during standardized contrast-enhanced PE CT: The first group was directed to inspire and hold their breath (apnea) during contrast medium injection. The second group was investigated during expiration followed by arrest of breathing. The third group performed Valsalva with a standardized pressure of $+20 \mathrm{mmHg}$. Finally, the fourth group was examined with suction against resistance, standardized to an underpressure of $-10 \mathrm{mmHg}$. The method is illustrated in Figs. 1 and 2.

\section{CT and contrast injection protocol}

All patients were scanned on the same CT system (iCT 256, Philips healthcare, Best, The Netherlands). All patients were investigated in the standardized supine position. Every subject had a venous access (Venflon 18 gauge venous access) over the cubital veins. Contrast medium (Iopromide DCI, Ultravist 300, Bayer Healthcare, Berlin, Germany) was administered intravenously using a contrast power injector (Stellant IPX 1, Medrad Company, Bayer Healthcare, Germany) with a weight-adapted contrast dose of $1 \mathrm{ml} / \mathrm{kg}$ at a rate of $4 \mathrm{cc} / \mathrm{sec}$. Saline flush $(30 \mathrm{ml})$ followed contrast medium injection with the same injection rate. In most cases, contrast medium was 
Fig. 1 Cross-section pulmonary CT images of four different exemplary cases demonstrating typical attentuation differences of the right lower pulmonary lobe artery in relation to studied breathing methods. Highest quantitative HU results (here 639 $\mathrm{HU}$ ) are observed in the "suction against resistance" / standardized Mueller manoeuvre group
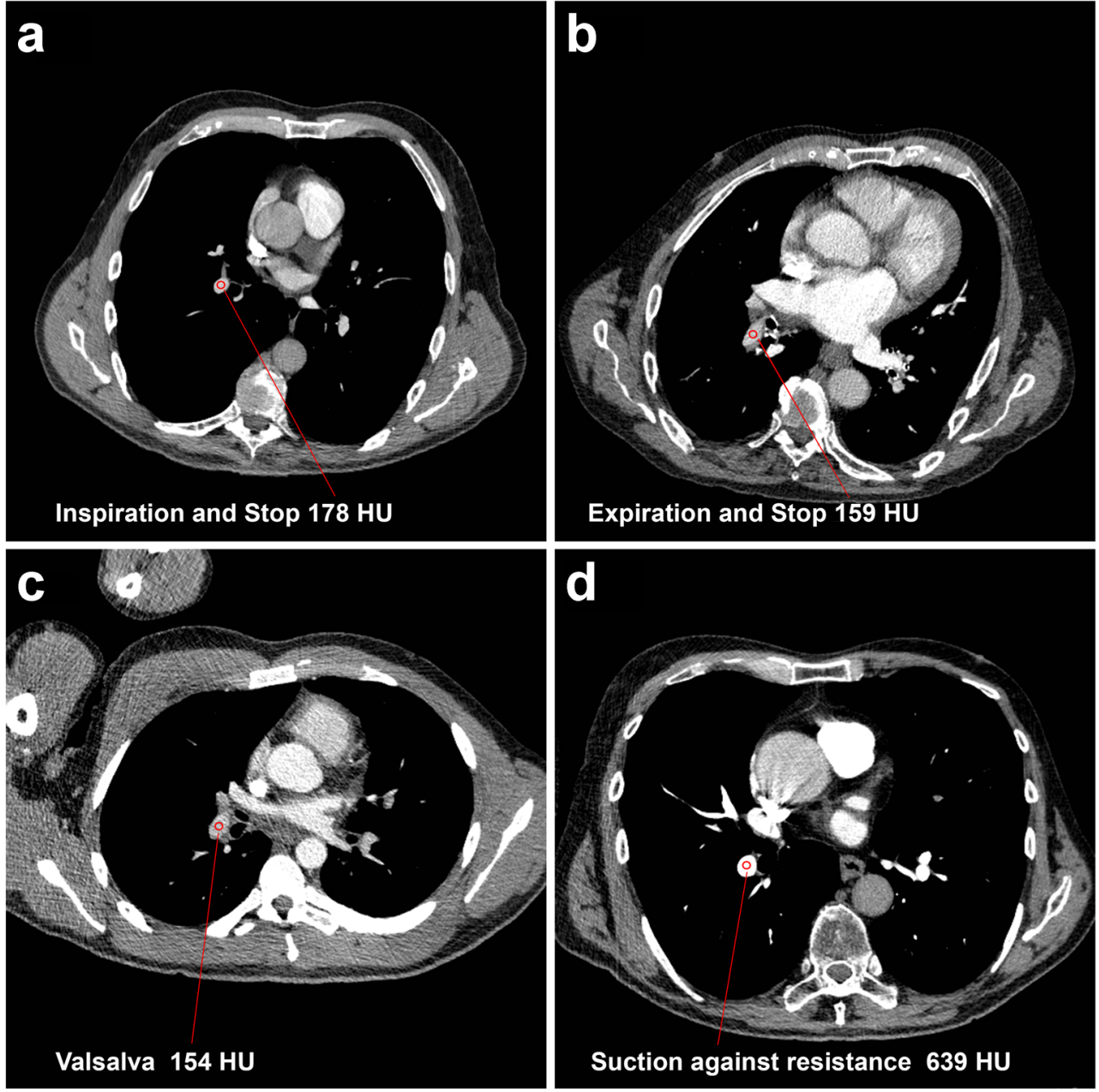

injected via the left antecubital vein (52 patients), but in eight cases, contrast medium was injected via the right antecubital vein because venous access was not successful on the left side.
The following technical parameters of the scan protocol were used for the PE CT: $128 \times 0.625 \mathrm{~mm}$ collimation, 0.993 pitch, $0.5 \mathrm{sec}$ rotation speed, $100 \mathrm{kVp}$ tube voltage and $200 \mathrm{mAs}$ tube current. The bolus-tracking technique with
Fig. 2 This image illustrates patient position inside the $\mathrm{CT}$ scanner during suction against resistance using the dedicated breathing device. The technique is described more in detail in recent publication [14]. Observing the manometer, the patient gets clear feedback and thus can control airway pressure

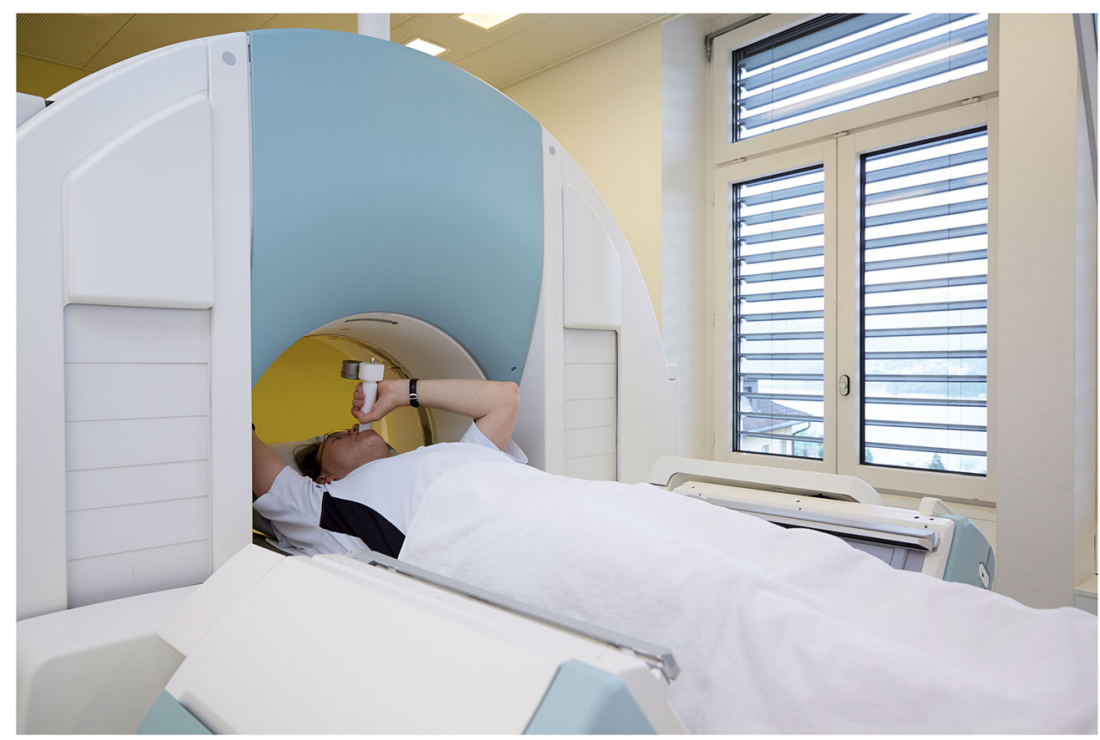


a region of interest placed in the center of the pulmonary trunk was used with a triggering threshold of $80 \mathrm{HU}$ and a delay of $5 \mathrm{sec}$ after reaching this cutoff value.

\section{Standardized suction and Valsalva maneuver with a dedicated CT manometer}

To perform standardized and reproducible Valsalva and suction maneuvers, we developed an easily readable manometer that could be used by the patients during the CT examination. A changeable disposable mouthpiece was fixed to one end of the plastic tube to ensure proper hygiene, while the contralateral side was connected to the pressure measurement device. The device could be used during application of the contrast medium and CT scan allowing self-control of the respiration pressure to perform a highly standardized and reproducible Valsalva maneuver with $+20 \mathrm{mmHg}$.

Moreover, for the "suction/inspiration against resistance" group, we provided a highly standardized and reproducible underpressure manometer permitting suction modulation at $10 \mathrm{mmHg}$ (Figs. 1 and 2). This technique has been described in detail in a recent publication [14].

\section{Standardized breathing maneuvers}

In all cases, the CT technician instructed the patient before the scan on how to perform the breathing maneuver. Before starting the dedicated breathing maneuver and during the initial phase of contrast medium administration, the patients were advised to follow a normal and regular breathing rhythm.

After reaching the predefined threshold value within the PA, patients of two groups were requested via the communication system to either inspire or otherwise expire followed by breath-hold (complete arrest of breathing) during contrast medium injection and ongoing pulmonary CT scan. Patients' compliance was assured by the supervising principal investigator, who controlled the abdomen movements during breath hold. According to our experience and knowledge, these two breathing maneuvers are the most commonly used methods to perform PE CT throughout the world in a normal clinical setup.

In the other two groups, patients were invited to perform a standardized manometer-assisted Valsalva or suction maneuver after reaching the predefined cutoff value within the PA. Therefore, the patient either started to push (Valsalva) or to suck air through the tube, with easily visible pressures on manometer providing direct feedback to the patient, permitting achievement of the predefined target pressures (Fig. 2).

The breathing maneuver was stopped after termination of the PE CT examination. Subsequently, the regular postvenous phase $\mathrm{CT}$ was started, to acquire diagnostic images following the regular protocol.

\section{Image analysis with quantification of Hounsfield units}

For further evaluation, source images were sent to a regular PACS system (Impax EE, Agfa Healthcare, Germany), where they were viewed using a standardized pulmonary angiographic window level (470 HU width, level $120 \mathrm{HU})$ on the originally acquired slice thickness of $1 \mathrm{~mm}$ (Fig. 1).

Quantitative measurements were performed in predefined locations: central part of the pulmonary trunk, central parts of the left and right pulmonary artery, left and right lower lobe pulmonary artery, and left and right lower lobe posterior segmental artery.

On each level, the size of the region of interest (ROI) was standardized: a ROI size of $200-300 \mathrm{~mm}^{2}$ was used within the pulmonary trunk, while a standardized ROI size of 40 to $60 \mathrm{~mm}^{2}$ was measured in the bilateral pulmonary artery. In the small segmental arteries, a standardized ROI size of 10 to $30 \mathrm{~mm}^{2}$ was used. The size of the ROIs was chosen in accordance with recent publications [8].

All patients were measured separately by two different, independent readers who were blinded with regard to the breathing maneuver used. One reader had 3 years of experience in reading pulmonary $\mathrm{CTs}(\mathrm{SW})$, while the other reader had 14 years of experience (principal investigator, AG). To guarantee that the second / principal investigator was definitely blinded, images were anonymized and were only read 20 weeks after finishing all inclusions.

\section{Statistical analysis}

The intraclass correlation coefficient (ICC) between the two readers was calculated. Reliability measurements were highly satisfactory (0.783-0.998 according to the various locations) so that the means of the two readers were used for further analysis. For particular sections, a general linear model (GLM) univariate analysis of variance with simple contrasts was performed. Thus, a GLM univariate analysis of variance was performed (a) for the estimated marginal means (for patient weight) of all pulmonary sections as a dependent variable; (b) for the central part of the pulmonary trunk by taking the estimated marginal means (for patient weight) of the right and left PA as a dependent variable; and (c) for the peripheral parts by taking the estimated marginal means (for patient weight) of the right and left lower lobe pulmonary and posterior segmental artery as a dependent variable. For all models, weight was included as a covariate. The significance level was set at a $p$ value of 0.05 .

Due to the fact that this study was a pilot study without previous experience within patients, the acquired data were used for a sample size calculation defining a power of $80 \%$ $(p<0.05)$ to demonstrate significant enhancement differences between the inspiration or expiration groups in comparison to 
Table 1 Descriptive characterization of the four patient groups (gender, age). No significant statistical difference between the pivot groups was found

\begin{tabular}{|c|c|c|c|c|c|}
\hline \multirow[t]{2}{*}{ Patient characteristics } & \multicolumn{4}{|l|}{ Protocol } & \multirow[t]{2}{*}{ p-value } \\
\hline & $+20 \mathrm{mmHg}$ Valsalva & $-10 \mathrm{mmHg}$ suction & Inspiration stop & Expiration stop & \\
\hline \multicolumn{6}{|l|}{ Sex } \\
\hline Male & $12(80.0 \%)$ & $11(73.3 \%)$ & $9(60.0 \%)$ & $9(60.0 \%)$ & 0.643 \\
\hline Female & $3(20.0 \%)$ & $4(26.7 \%)$ & $6(40.0 \%)$ & $6(40.0 \%)$ & \\
\hline \multicolumn{6}{|l|}{ Age } \\
\hline Mean & 66.4 & 69.4 & 64.7 & 63.4 & 0.496 \\
\hline Standard Deviation & 13.6 & 12.0 & 8.8 & 9.4 & \\
\hline Weight (mean kg, \pm standard deviation) & $75.7( \pm 10.9)$ & $69.4( \pm 12.7)$ & $71.7( \pm 19.8)$ & $72.9( \pm 13.1)$ & 0.686 \\
\hline
\end{tabular}

the suction group. Descriptive statistics were used for age, gender and weight comparison to exclude group differences.

\section{Results}

Overall, 60 patients were successfully included, covering all pulmonary vessels of interest. There were no statistically significant differences between the clinical descriptive characteristics of age $(p=0.496)$, gender $(p=0.643)$, and weight ( $p=$ 0.686 ) between the four different groups (Table 1).

\section{Statistical comparison of contrast density of standardized breathing maneuvers}

a) Comparison of contrast density of suction against other breathing maneuvers within all pulmonary vessels

The observed pulmonary vascular enhancement characteristics of every group covering all previously defined vascular regions are summarized in Table 2 and Fig. 3. It could be shown that in all vascular regions, contrast density was superior during suction against resistance in comparison with other breathing techniques. Overall, a superior mean difference of $119.96 \mathrm{HU}(p=0.002)$ compared to Valsalva was recorded. Moreover, a mean density of $60.76 \mathrm{HU}(p=0.11)$ compared to inspiration and a mean density of $21.98 \mathrm{HU}(p=0.56)$ in comparison to expiration were recorded in favour of suction mode.

Table 2 Contrast attenuation results for all mean densities of suction against resistance in comparison to other breathing methods for all parts of the pulmonary artery system (pulmonary trunk, right and left pulmonary artery, right and left lower lobe pulmonary artery, and right b) Comparison of contrast density of suction against all other breathing maneuvers within the central vessels of the PAs

The observed central parts of the pulmonary trunk are summarized in Table 3 and Fig. 4. It could be demonstrated that contrast enhancement was superior during suction against resistance mode. Indeed, measured mean contrast densities were $95.74 \mathrm{HU}$ superior $(p=0.020)$ to Valsalva, $51.15 \mathrm{HU}$ superior $(p=0.201)$ to inspiration, and 10.61 HU superior $(p=0.79)$ to expiration, respectively.

c) Comparison of contrast density of suction against other breathing maneuvers within the peripheral parts of the PA system

The assessed peripheral parts of pulmonary vascular enhancement are summarized in Table 4 and Fig. 5. It could be shown that in all vascular regions of the peripheral parts, attenuation was superior during suction against resistance compared to the other breathing methods. The enhancement was $138.14 \mathrm{HU}$ superior $(p=0.001)$ to Valsalva, 67.97 HU superior ( $p=0.075)$ to inspiration and $30.51 \mathrm{HU}$ superior $(p=0.420)$ to expiration, respectively.

\section{Discussion}

The presented study results demonstrate that overall, suction against resistance yields the highest contrast density within the

and left lower lobe pulmonary posterior segmental pulmonary artery). Reported values are estimated marginal means for patient weight. Patient weight was included as a covariate in the GLM

\begin{tabular}{lllll}
\hline Breathing method versus & & Mean difference & $p$ value & $95 \%$ CI \\
\hline-10 mmHg suction & +20 mmHg Valsalva & 119.96 & 0.002 & $44.14-195.79$ \\
& Inspiration stop & 60.76 & 0.11 & $-14.24-135.76$ \\
& Expiration stop & 21.98 & 0.560 & $-53.19-97.16$ \\
\hline
\end{tabular}


Fig. 3 Radio-density (HU) mean of the whole pulmonary vessel system: pulmonary trunk, right and left pulmonary artery, right and left lower lobe pulmonary artery, and right and left lower lobe pulmonary posterior segmental pulmonary artery and breathing methods $+20 \mathrm{mmHg}$ Valsalva (1), -10 mmHg suction (2), inspiration stop (3), and expiration stop (4). Boxes show the median and the 25th and 75th percentile, whiskers show 10th and 90th percentile

\begin{tabular}{lcccccccc}
\hline $\begin{array}{l}\text { Breathing } \\
\text { method }\end{array}$ & N & Minimum & Maximum & Mean & SD & Median & $\mathbf{Q}_{1}$ & $\mathbf{Q}_{3}$ \\
\hline $\begin{array}{l}+20 \mathrm{mmHg} \\
\text { Valsalva }\end{array}$ & 15 & 157.50 & 541.14 & 298.43 & 109.48 & 279.79 & 205.21 & 352.14 \\
$-10 \mathrm{mmHg}$ & 15 & 312.71 & 636.29 & 431.99 & 92.57 & 423.07 & 356.00 & 482.64 \\
suction & & & & & & & & \\
Inspiration stop & 15 & 178.50 & 518.71 & 366.36 & 103.11 & 330.14 & 298.57 & 456.29 \\
Expiration stop & 15 & 157.07 & 575.93 & 402.42 & 117.56 & 380.79 & 324.29 & 530.93 \\
\hline
\end{tabular}

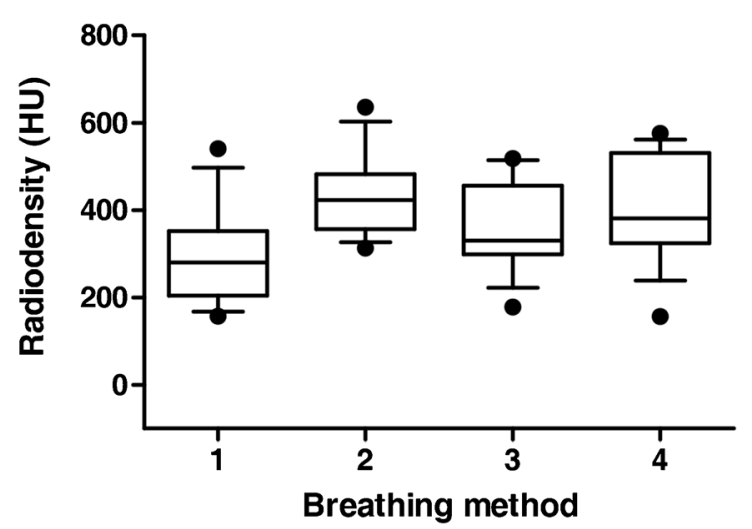

PAs in comparison to all other breathing maneuvers. Moreover, in the more critical peripheral parts of the pulmonary artery system, this advantage was even more pronounced (suction versus Valsalva with $+138.14 \mathrm{HU} ; p=0.001$; suction versus expiration with $+30.51 \mathrm{HU} ; p=0.420$; suction versus inspiration with $+67.51 \mathrm{HU} ; p=0.075$, respectively). It should be emphasized that patient weight could be ruled out as an explanatory factor for these surprising results. Evidently, the pivot group included in this study was too small to reach statistical significance for all breathing maneuvers. Ethical considerations concerning the pilot-study design and increased patient radiation exposure limited the size of the study group.

Optimal enhancement levels for pulmonary CT to detect embolism and ideal injection protocols are still under debate [15]. Especially the peripheral parts of the PA require optimal contrast enhancement to achieve high sensitivity for detecting of PE [16]. In recently published data, contrast densities within the peripheral parts of the pulmonary arteries using various optimized protocols were reported to be between 370 and 385 HU. Our data exceed these values when using "suction against resistance" breathing maneuver (mean $=434 \mathrm{HU}$; maximum =

Table 3 Contrast attenuation results for mean densities of suction against resistance in comparison to other breathing methods of central parts of the pulmonary artery system (pulmonary trunk, right and left

\begin{tabular}{lllll}
\hline Breathing method versus & & Mean difference & $p$ value & $95 \%$ CI \\
\hline-10 mmHg suction & +20 mmHg Valsalva & 95.74 & 0.02 & $15.61-175.87$ \\
& Inspiration stop & 51.15 & 0.201 & $-28.11-130.40$ \\
& Expiration stop & 10.61 & 0.790 & $-68.83-90.05$ \\
\hline
\end{tabular}

$637 \mathrm{HU})$. It should be noted that our inspiration and expiration contrast density data corroborate those of this previous study, measured using a breathhold technique [17]. However, it should be emphasized that the influence of the breathing technique was not thoroughly addressed in this earlier study.

Numerous publications conducted over the last few years have discussed the influence of different breathing maneuvers on the quality and enhancement profiles of PE CT. Despite occasional controversial discussions, there was a general consensus that expiration followed by breathhold leads to an improved contrast in pulmonary arteries in comparison to inspiration followed by breathhold $[8-13,18]$. To our knowledge, the present prospective clinical study is the first to investigate on a patient level how the recently introduced and promising "suction against resistance" breathing maneuver might influence contrast behavior compared to other breathing techniques used in clinical routine [14]. Indeed, in comparison to previous $\mathrm{CT}$ studies including a larger cohort and comparing inspiration versus expiration breathing maneuvers, our absolute $\mathrm{CT}$ attenuation levels exceed these previously reported results. In fact, in our own study group, suction against resistance leads to more elevated mean attenuation values (432 pulmonary artery). Reported values are estimated marginal means for patient weight. Patient weight was included as a covariate in the GLM 
Fig. 4 Radio-density (HU) of the central parts of the pulmonary vessel system: mean of pulmonary artery and breathing methods $+20 \mathrm{mmHg}$ Valsalva (1), $-10 \mathrm{mmHg}$ suction (2), inspiration stop (3), and expiration stop (4). Boxes show the median and the 25th and 75 th percentile, whiskers show 10 th and 90 th percentile pulmonary trunk, right and left

\begin{tabular}{lcccccccc}
\hline $\begin{array}{l}\text { Breathing } \\
\text { method }\end{array}$ & $\mathbf{N}$ & Minimum & Maximum & Mean & SD & Median & $\mathbf{Q}_{1}$ & $\mathbf{Q}_{3}$ \\
\hline $\begin{array}{l}+20 \mathrm{mmHg} \\
\text { Valsalva }\end{array}$ & 15 & 158.67 & 549.83 & 319.86 & 126.49 & 320.67 & 195.00 & 397.83 \\
$\begin{array}{l}-10 \mathrm{mmHg} \\
\text { suction }\end{array}$ & 15 & 316.67 & 643.83 & 429.43 & 87.93 & 405.83 & 347.33 & 483.17 \\
$\begin{array}{l}\text { Inspiration } \\
\text { stop }\end{array}$ & 15 & 188.17 & 563.00 & 373.33 & 109.80 & 343.17 & 300.50 & 458.33 \\
\begin{tabular}{l} 
Expiration stop \\
\hline
\end{tabular} & 15 & 161.67 & 586.17 & 411.10 & 119.00 & 385.67 & 340.83 & 561.00 \\
\hline
\end{tabular}

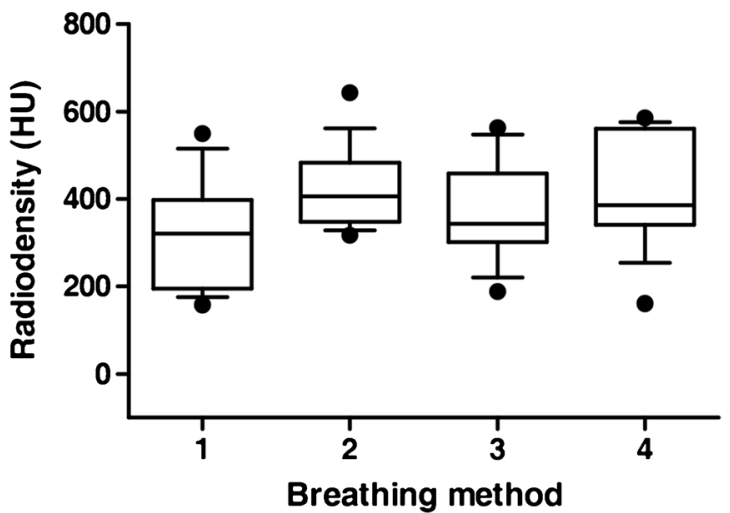

HU) in all pulmonary vessels compared to Valsalva, inspiration and expiration (between 298 and 402 HU according to the different breathing maneuvers). Furthermore, suction against resistance also leads to increased attenuation levels in comparison to previous CT studies (inspiration from 243 to 282; expiration from 265 to $296 \mathrm{HU}$ ) [8]. These contrast density differences indicate that breathing techniques bear an important influence on contrast density behaviour.

To improve reproducibility and for standardization purposes, especially during suction and Valsalva, we used a dedicated, newly designed manometer system to achieve an air pressure controlled breathing maneuver from the patient. This technique has been recently described in more detail [14]. It should be emphasized that despite suspicious PE, advanced age or general diseases, all patients were able to comply with the requested breathing maneuver.

Reflecting our results, the question is, what might be the physiological explanation for the observed highest contrast with suction against resistance breathing maneuver? As described graphically in Fig. 6 (and Video 1, supplementary material), suction against resistance exerts a specific

Table 4 Contrast attenuation results for mean densities of suction against resistance in comparison to other breathing methods of peripheral parts of the pulmonary artery system (right and left lower lobe pulmonary artery, and right and left lower lobe pulmonary

\begin{tabular}{llccc}
\hline Breathing method versus & & Mean difference & $p$ value & $95 \%$ CI \\
\hline-10 mmHg suction & +20 mmHg Valsalva & 138.14 & 0.001 & $62.18-214.09$ \\
& Inspiration stop & 67.97 & 0.075 & $-7.15-143.10$ \\
& Expiration stop & 30.51 & 0.42 & $-44.79-105.81$ \\
\hline
\end{tabular}

physiological influence on the flow ratios of the superior und inferior vena cava, as described recently [14, 19]. First, during normal inspiration without any resistance, the diaphragm moves caudally. As a result of this strong distinct caudal movement of the diaphragm, the abdominal organs are displaced ventrally and dorsally. One can observe this effect by placing a hand on the abdominal wall during inspiration and noticing how the abdominal wall bulges outwards. Due to this displacement of the abdominal organs, pressure forms directly on the IVC. Ultimately, this causes a strong inflow of non-contrasted blood from the lower half of the body into the right atrium. During such a phase, the iodinecontaining contrast medium flows into the right side of the heart via the arm vein and the superior vena cava, on account of the non-contrasted blood from the inferior vena cava. This leads to stronger dilution due to the mixing of the contrasted and non-contrasted blood. Such a phenomenon is also called transient interruption of contrast bolus, potentially causing attenuation decrease within the PA [10].

Secondarily, in contrast to simple inspiration, in the case of suction against resistance, strong tension develops in the

posterior segmental pulmonary artery). Reported values are estimated marginal means for patient weight. Patient weight was included as a covariate in the GLM 
Fig. 5 Radio-density (HU) of the peripheral parts of the pulmonary vessel system: mean of right and left lower lobe pulmonary artery, and right and left lower lobe pulmonary posterior segmental pulmonary artery and breathing methods $+20 \mathrm{mmHg}$ Valsalva (1), $-10 \mathrm{mmHg}$ suction (2), inspiration stop (3), and expiration stop (4). Boxes show the median and the 25th and 75th percentile, whiskers show 10th and 90th percentile

\begin{tabular}{lcccccccc}
\hline $\begin{array}{l}\text { Breathing } \\
\text { method }\end{array}$ & $\mathbf{N}$ & Minimum & Maximum & Mean & SD & Median & $\mathbf{Q}_{1}$ & $\mathbf{Q}_{3}$ \\
\hline $\begin{array}{l}+20 \mathrm{mmHg} \\
\text { Valsalva }\end{array}$ & 15 & 156.63 & 534.63 & 282.37 & 103.81 & 248.75 & 216.63 & 333.25 \\
$\begin{array}{l}-10 \mathrm{mmHg} \\
\text { suction }\end{array}$ & 15 & 309.75 & 636.88 & 433.90 & 99.24 & 441.00 & 352.25 & 482.25 \\
Inspiration stop & 15 & 171.25 & 525.63 & 361.13 & 102.57 & 325.13 & 278.38 & 437.75 \\
Expiration stop & 15 & 153.63 & 568.25 & 395.92 & 117.85 & 377.13 & 311.88 & 506.63 \\
\hline
\end{tabular}

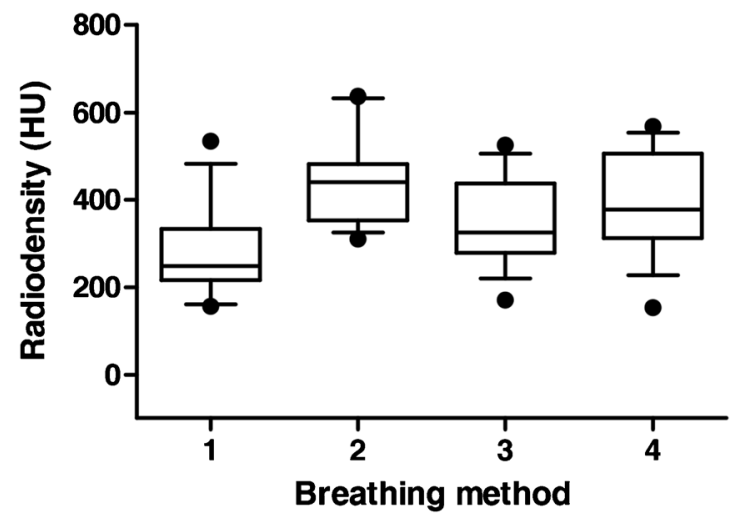

\section{Suction against Resistance}
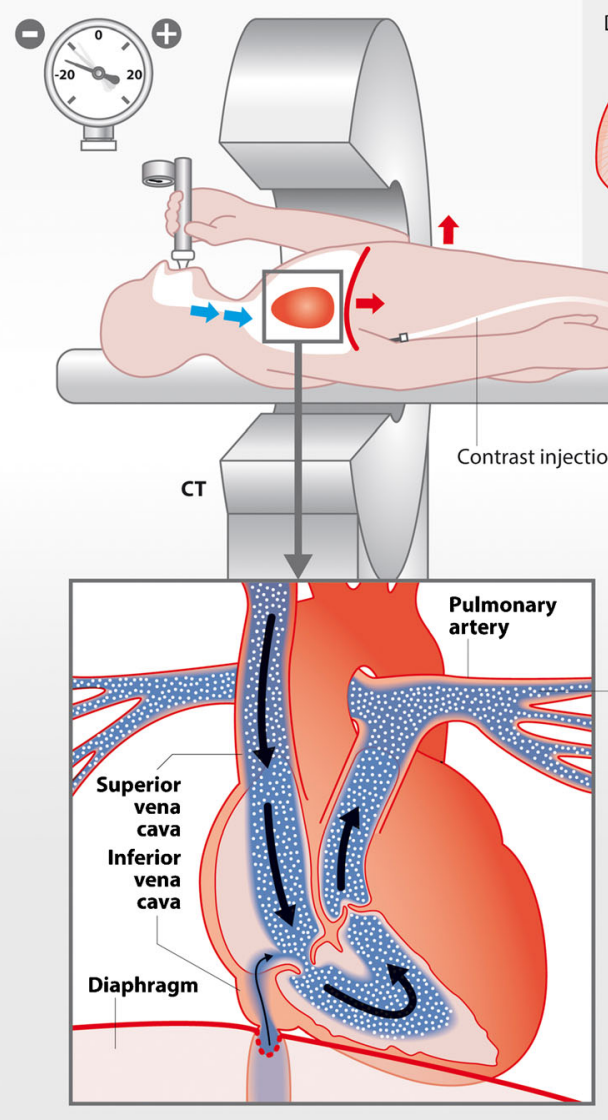

Fig. 6 This schematic diagram is intended to show exemplarily what causes the effect of suction against resistance compared with the normal inspiration and stop. In the case of suction against resistance, a strong muscular contraction of the diaphragm occurs, causing a collapse and almost complete occlusion of the IVC. Simultaneously, as a result of

\section{Inspiration and Stop}

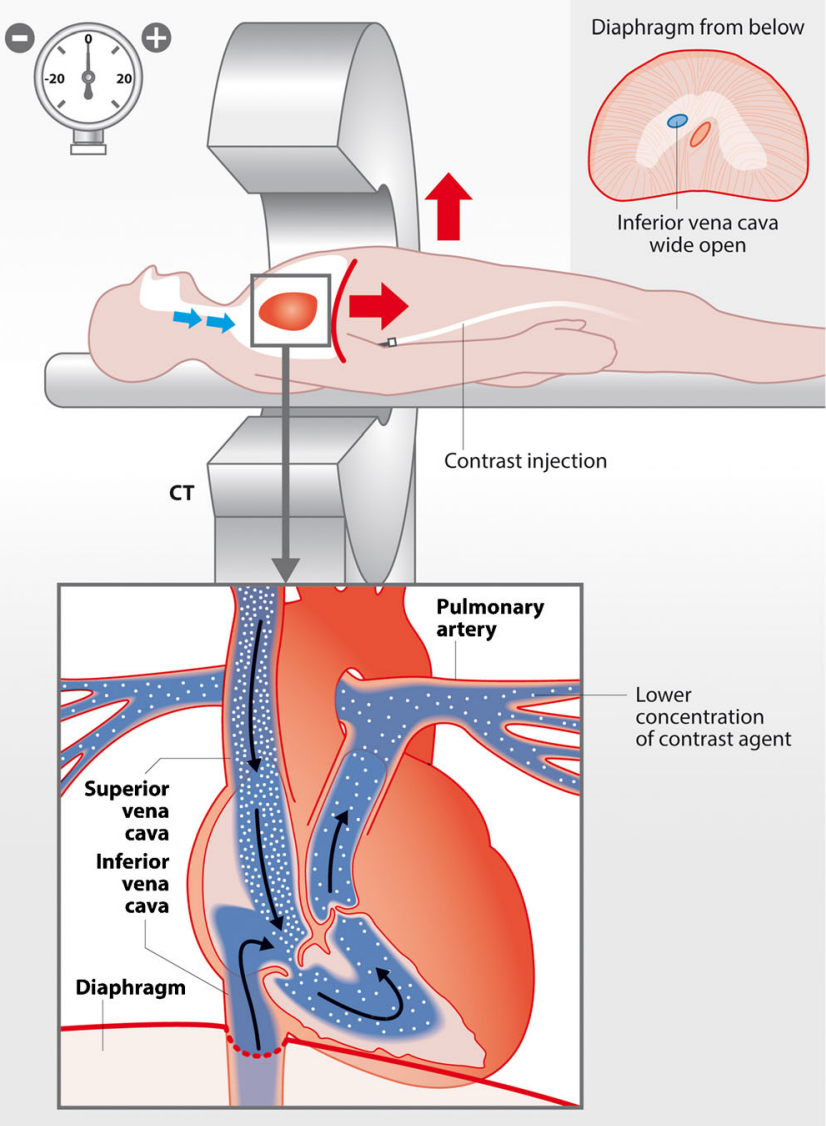

caudal movement of the diaphragm, a suction effect on the SVC occurs. As a result, the inflow of the contrasted SVC blood and non-contrasted IVC blood flow is minimized, leading to a more homogenous and concentrated contrast in the pulmonary artery 
diaphragm. Due to the under-pressure, the diaphragm moves markedly less caudally, resulting in the abdominal organs hardly being displaced at all. One can observe this phenomenon on oneself by attempting to inspire with mouth and nose closed as in the Mueller maneuver. The reader will notice that the abdominal wall practically does not bulge out at all. Simultaneously, due to the strong muscular contraction of the diaphragm, a slot-shaped narrowing of the inferior vena cava occurs, which additionally prevents the inflow of the non-contrasted blood on the abdominal cavity via the inferior vena cava (Fig. 6, Video 1). The collapsing of the IVC during the Mueller maneuver was already described in 1968 [20]. In our opinion, this is a very good explanation of why flow via the IVC during this maneuver is so strongly reduced, while blood flow via the SVC is significantly increased in order to compensate the reduction from beyond. This corroborates our previous volunteer-based results with distinct phase-contrast flux and volume measurements of both vascular regions [14].

It should be critically remarked that the study bears several limitations. Firstly, as explained above, the inclusion number was too small to reach statistical significance in all cases. Our patient number of 15 in each group was smaller than those of previous $\mathrm{CT}$ studies comparing various breathing techniques (from 145 to 327 patient inclusions) [7, 8]. Although these previous data had been submitted to our Ethical Committee, a higher number of patients was denied due to pilot study design. Secondly, the start of the scan triggered by an ROI in the main trunk of the pulmonary artery might have led to a rather late start of the breathing maneuvers. This assumption needs to be further clarified in future clinical studies with a greater number of patients. Ultimately, the clinical potential of this new breathing maneuver for detecting pulmonary embolism in patients suffering symptoms of lung embolism needs to be confirmed.

\section{Conclusion}

In this pilot study, we show that the absolute attenuation values of PE CT under the breathing maneuver "suction against resistance" or the standardized Mueller maneuver are superior in comparison to other clinically established breathing maneuvers, in particular in the more peripheral PAs.

Acknowledgments We thank Professor Dr. med. Georg Bongartz from the Department of Radiology and Nuclear Medicine of the University Hospital in Basel (Switzerland) for his valuable input during the ECR 2012 lecture concerning influencing factors regarding contrast enhancement using pulmonary CT. This served as the final impetus to perform this study.

We would especially like to thank Martin Hinnen (Head of the Technicians' Service) and his CT Team of the Cantonal Hospital Winterthur for helping us manage and examine the patients.

Finally we want to thank Nicole Graf, who performed the statistical analysis (www.biostatistics.ch).
The scientific guarantor of this publication is Andreas Gutzeit. The authors of this manuscript declare relationships with the following companies: Author No. 2 is a consultant for Guerbet contrast media company. The authors of this manuscript declare no relationships with any companies, whose products or services may be related to the subject matter of the article. The authors state that this work has not received any funding. Several authors are experienced in statistics. No complex statistical methods were necessary for this paper. Institutional Review Board approval was obtained. Written informed consent was obtained from all subjects (patients) in this study. Methodology: prospective, randomized controlled trial, performed at one institution.

\section{References}

1. Bělohlávek J, Dytrych V, Linhart A (2013) Pulmonary embolism, part I: Epidemiology, risk factors and risk stratification, pathophysiology, clinical presentation, diagnosis and nonthrombotic pulmonary embolism. Exp Clin Cardiol 18:129-138

2. Rosso J, Gauthier H et al (2000) Systematic lung scans reveal a high frequency of silent pulmonary embolism in patients with proximal deep venous thrombosis. Arch Intern Med 160:159-164

3. Pineda LA, Hathwar VS, Grant BJ (2001) Clinical suspicion of fatal pulmonary embolism. Chest 120:791-795

4. Henzler T, Barraza JM Jr, Nance JW Jr et al (2011) CT imaging of acute pulmonary embolism. J Cardiovasc Comput Tomogr 5:3-11

5. Stein PD, Kayali F, Hull RD (2007) Spiral computed tomography for the diagnosis of acute pulmonary embolism. Thromb Haemost 98:713-720

6. Rathbun SW, Raskob GE, Whitsett TL (2000) Sensitivity and specificity of helical computed tomography in the diagnosis of pulmonary embolism: a systematic review. Ann Intern Med 132:227-232

7. Rassner UA, Thieszen SL, Phillips J, Oki A (2004) Contrast dynamics during CT pulmonary angiogram: analysis of an inspiration associated artifact. J Thorac Imaging 19:1-7

8. Mortimer AM, Singh RK, Hughes J, Greenwood R, Hamilton MC (2011) Use of expiratory CT pulmonary angiography to reduce inspiration and breath-hold associated artefact: contrast dynamics and implications for scan protocol. Clin Radiol 66:1159-1166

9. Chen YH, Velayudhan V, Weltman DI, Balsam D, Patel N, Draves $\mathrm{KA}$ et al (2008) Waiting to exhale: salvaging the nondiagnostic CT pulmonary angiogram by using expiratory imaging to improve contrast dynamics. Emerg Radiol 15:161-169

10. Wittram C, Yoo AJ (2007) Transient interruption of contrast on CT pulmonary angiography: proof of mechanism. J Thorac Imaging 22:125-129

11. Arenas-Jiménez J, Bernabé-García J, García-Espasa C (2013) Re: Use of expiratory CT pulmonary angiography to reduce inspiration and breath-hold associated artefact: contrast dynamics and implications for scan protocol. Clin Radiol 68:e 98

12. Hamilton MC, Mortimer AM, Hughes J (2013) Re: Use of expiratory CT pulmonary angiography to reduce inspiration and breathhold associated artefact: contrast dynamics and implications for scan protocol. A reply. Clin Radiol 68:e99-e100

13. Bernabé-García JM, García-Espasa C, Arenas-Jiménez J, SánchezPayá J, de la Hoz-Rosa J, Carreres-Polo JO (2012) Has "respiratory coaching" before deep inspiration an impact on the incidence of transient contrast interruption during pulmonary CT angiography? Insights Imaging 3:505-511

14. Gutzeit A, Roos JE, Hergan K et al (2014) Suction against resistance: a new breathing technique to significantly improve the blood flow ratio of the superior and inferior vena cava. Eur Radiol 24: 3034-3041 
15. Lee CW, Seo JB, Song JW, Kim MY, Lee HY, Park YS et al (2011) Evaluation of computer-aided detection and dual energy software in detection of peripheral pulmonary embolism on dual-energy pulmonary CT angiography. Eur Radiol 21:54-62

16. Chen YH, Velayudhan V, Weltman DI et al (2008) Waiting to exhale: salvaging the nondiagnostic CT pulmonary angiogram by using expiratory imaging to improve contrast dynamics. Emerg Radiol 15:161-169

17. Ramadan SU, Kosar P, Sonmez I, Karahan S, Kosar U (2010) Optimisation of contrast medium volume and injection-related factors in CT pulmonary angiography: 64-slice CT study. Eur Radiol 20:2100-2107
18. Kuzo RS, Pooley RA, Crook JE, Heckman MG, Gerber TC (2007) Measurement of caval blood flow with MRI during respiratory maneuvers: implications for vascular contrast opacification on pulmonary CT angiographic studies. AJR Am J Roentgenol 188:839842

19. Fasshauer M, Joseph AA, Kowallick JT et al (2014) Real-time phase-contrast flow MRI of haemodynamic changes in the ascending aorta and superior vena cava during Mueller manoeuvre. Clin Radiol 69:1066-1071

20. Bergel DH, Gabe IT, Makin GS, Mills CJ (1968) Velocity of blood flow in normal human venae cavae. Circ Res 23:349-359 